\title{
Proceso de administración, producción y comercialización de queso en ASOPASN en el año $2013^{1}$
}

\author{
Process management, production and marketing of cheese in ASOSPAN 2013
}

\author{
Adriana Yamileth Castillo² \\ Jolimar Maykeling Gámez Osegueda ${ }^{3}$ \\ Belkys Karina Guevara Cruz ${ }^{4}$ \\ Beverly Castillo Herrera ${ }^{5}$
}

1 Este artículo se basa en la investigación titulada Proceso de Administración, Producción y Comercialización de queso en Asociación de Programas Agrícolas de San Nicolás (ASOPASN) en el año 2013. para optar al título de Licenciatura en Administración de Empresas por la UNAN-Managua, FAREM-Estelí.

2 UNAN-Managua, FAREM-Estelí. Correo electrónico: adcastillo89@yahoo.com

3 UNAN-Managua, FAREM-Estelí. Correo electrónico: yoli.gamez33@yahoo.es

4 UNAN-Managua, FAREM-Estelí. Correo electrónico: karbel.cruz@gmail.com

5 Master en Ciencias Sociales por la Universidad de Guadalajara, estudiante del Doctorado en Ciencias Sociales de la Universidad del Zulia. Docente titular de la UNAN-Managua, FAREM-Estelí. Correo electrónico: beverly.castillo@yahoo.com.

\section{RESUMEN}

En el presente estudio investigativo se realizó una evaluación del proceso de administración, producción y comercialización de queso de la asociación de programas agrícolas de san Nicolás (ASOPASN) en el año 2013. La investigación es del tipo cualitativo porque aborda a profundidad la problemática de Administración, producción y comercialización de queso en ASOPASN, para ello se hace una descripción holística y se analiza exhaustivamente el problema para sugerir una estrategia que mejore su funcionamiento. El universo de estudio estuvo conformada por la tienda la Garnacha, proyecto perteneciente a la Asociación de Programas Agrícolas de San Nicolás (ASOPANS) en el municipio de Estelí. Dentro de las mejoras que se deben hacer en esta asociación, está el seguimiento al proceso de administración en las principales actividades que se realizan, en el proceso de producción velar por el cumplimiento de las buenas prácticas de manufactura (BPM), así mismo en el proceso de comercialización se debe de garantizar el cumplimiento de las normas exigidas por el ministerio de salud (MINSA) para que de esta manera se pueda obtener la renovación del registro sanitario que actualmente se encuentra suspendido por el incumplimiento de algunos requisitos establecidos por el ministerio de salud.

Palabras claves: Proceso, Administración, Producción, Comercialización.

\section{ABSTRACT \\ In this research study a process evaluation of management, production and marketing of cheese association of the agricultural programs of St. Nicholas (ASOPASN) in 2013 was performed. The research is a qualitative approach that addresses in depth the problem of administration, production and marketing}


of cheese ASOPASN, for it takes a holistic description and the problem was exhaustively analyzed to suggest a strategy to improve its performance. The study group consisted of the store LaGarnacha project belonging to the Association of Agricultural Programs San Nicolas (ASOPANS) in the municipality of Estelí. Among the improvements to be made in this association is monitoring the management process in the main activities conducted in the production process to ensure the fulfillment of the good manufacturing practices (GMP), also in the marketing process must guarantee compliance with the standards required by the Ministry of Health (MINSA) so that in this way you can get the renewal of the health record that is currently suspended for failure to comply with certain requirements set by the Ministry of health.

\section{INTRODUCCIÓN}

La Asociación de Programas Agrícolas de San Nicolás (ASOPASN) durante el transcurso de los años ha promovido el desarrollo integral de las familias rurales y facilitado procesos para fortalecer las capacidades productivas, organizativas, de gestión y alternativas para diversificar la producción en la búsqueda de la sostenibilidad económica.

Dicha asociación alcanza su estabilidad económica mediante emprendurismo de negocios desarrollado por los socios y habitantes de la comunidad de san Nicolás, dentro de este contexto encontramos la tienda la Garnacha que resulta ser la Finca de esta asociación con un gran potencial turístico y productivo.

La tienda está ubicada en el municipio de san Nicolás en el departamento de Estelí, formando parte del centro turístico la garnacha el cual tiene reconocimiento a nivel nacional e internacional ya que cuenta con una riqueza en flora y Fauna.

En esta tienda se comercializa artesanía, miel, plantas medicinales, queso de cabra y vaca, siendo estos últimos los puntos de atracción de los turistas ya que es un queso diferente al que tradicionalmente se conoce, por los métodos suizos utilizados en la producción.

Entre las limitantes existentes en el proceso de administración de la Asociación de Programas Agrícolas de San Nicolás (ASOPASN) encontramos la falta del seguimiento administrativo a las actividades desarrolladas por esta asociación, actualmente este proceso solo se da una vez por semana, careciendo de control y formalidad empresarial.

Una de las limitantes de la producción de queso es la escasez de la materia prima ya que la Asociación trabaja con los acopios aledaños a la zona de san Nicolás y con las cabras y vacas con las que cuentan como asociación siendo en total 25 cabras y 30 vacas recolectando y procesando un promedio de 250 Its de leche semanal incluida leche de cabra y vaca.

La libre comercialización de queso en esta asociación se ha visto obstaculizada a consecuencia del vencimiento del registro sanitario ya que cuando el Ministerio de Salud (MINSA) ha realizado las revisiones sanitarias ha considerado que la infraestructura es inadecuada para la producción de queso, por lo tanto no se ha podido renovar el registro sanitario, aparte de esto se ha mostrado un gran desinterés de parte de los socios de ASOPASN de presentarse al ministerio de salud de Managua, a cumplir con los requisitos y el análisis necesarios de sus productos alimenticios.

A consecuencia de esta situación el convenio de distribución de queso que existía entre ASOPASN y el supermercado la Colonia se vio truncada, actualmente no se permite el abastecimiento de queso por la falta del registro sanitario.

También se carece de personal capacitado para el manejo de las cabras que tengan conocimiento sobre la alimentación y cuido de las cabras productoras. 
El presente trabajo investigativo procura describir el proceso administrativo y productivo implementado por la asociación de programas agrícolas de san Nicolás, así como analizar las estrategias de comercialización utilizadas por ASOPASN en la distribución de queso suizo en el mercado diferenciado.

El centro de atención de la investigación es la producción y comercialización de queso de cabra y vaca ya que estos son elaborados mediante métodos suizos que han creado una gran diferencia en cuanto a los quesos que tradicionalmente se consumen. El método suizo ha llegado a ser aplicado por los trabajadores de Asopasn gracias a las capacitaciones a las que se sometió el técnico de dicho lugar y las que fueron impartidas por un conocedor del método suizo de nacionalidad italiana.

La realización de este trabajo será de gran utilidad para ASOPAN ya que les permite profundizar mejor en la realidad de su proceso productivo, y conocer la preferencia de su mercado diferenciado para tomar decisiones administrativas considerando las estrategias sugeridas en este estudio. Así mismo La investigación será de beneficio para las instancias que manejan el Área Protegida del TISEY les permitirá reconocer una experiencia de manejo adecuado y amigable de la producción agropecuaria con el medio ambiente.

\section{MATERIALES Y MÉTODOS}

La presente investigación es aplicada ya que estudia y analiza un problema real con el propósito de plantear propuestas para mejorar el funcionamiento de ASOPASN. Así mismo es del tipo cualitativa porque aborda a profundidad la problemática de administración, producción y comercialización de queso.

Este es una investigación con muestra No Probabilística, de tipo causal porque no todo el universo tiene la probabilidad de ser seleccionado. Dentro de los criterios de selección de la muestra están: 1) Disposición de tiempo del Propietario y los trabajadores para colaborar con la investigación; 2) Personas involucradas en el proceso de Producción Y Comercialización de queso; y 3) Experiencia de 2 a más años en el área productiva y de Comercialización.

Para el cumplimiento de los objetivos que se plantean en esta investigación se utilizaron tres técnicas de recolección de datos: la investigación documental, la entrevista semi-estructurada y la guía de observación.

La Entrevista se aplicó a personas claves, tal es el caso de los socios de la ASOPASN cuya información nos permitió conocer más de esta asociación y los rubros a los cuales se dedican, así mismo se aplicó entrevista a los propietarios de los negocios en donde se comercializa este queso, de igual forma se entrevistó a funcionarios del SILAIS ESTELI en representación del MINSA de Managua que nos proporcionó la información necesaria para conocer la situación actual de ASOPASN en cuanto al Registro Sanitario.

La guía de observación se aplicó en dos lugares, primero se realizó la observación en el área de producción para conocer el flujo productivo de este queso mediante métodos suizos. También se realizó la observación en el supermercado la Segovia para conocer la demanda del queso e identificar a los clientes potenciales del mismo.

\section{RESULTADOS Y DISCUSIÓN}

Caracterización de la Empresa ASOPASN del Municipio de San Nicolás de la Ciudad de Estelí

La Asociación Programa Agrícola San Nicolás (ASOPASN) se constituyó legalmente en el 2001 con 250 socios de comunidades del municipio de San Nicolás, y hasta hoy es la única organización 
de productores que existe en el municipio aparte de la UNAG. La Asociación trabaja para fortalecer las capacidades productivas de los productores, contribuir a mejorar la seguridad alimentaria de las familias campesinas, buscar mercados buenos y estables para gestionar proyectos a los asociados. Tiene una finca integral donde desarrolla la agricultura orgánica con ganado bovino, cabras, ovejas y hortalizas.

Los últimos dos años la asociación ha hecho un esfuerzo para desarrollar el ecoturismo en La Garnacha con apoyo de PNUD tienen cabañas, rutas turísticas, un comedor y una venta de artesanía y productos propios de ASOPASN. La meta es un desarrollo integral económico y social en las comunidades asociadas.

ASOPASN es una asociación abierta. No 'amarra' a sus socios con convenios de venta. Cada socio tiene la libertad de vender sus productos a quien quiera. Todos están claros que para vender a la asociación tienen que cumplir con las normas de calidad establecidas para la producción orgánica. Los productores más cercanos a la comunidad de la Garnacha venden a la asociación y los que están más alejados venden de forma individual y local. Hace 5 años la mayoría de los productores se dedicaban a la agricultura convencional, usando químicos de alta peligrosidad. En horticultura, el repollo constituía la mayor parte de la producción, pero la producción no estaba dimensionada a la demanda del mercado. A veces el precio era tan bajo, que no les quedaba otra opción que usar el repollo como alimento para sus animales. En el 2002 la asociación empezó a experimentar con la agricultura orgánica en $1 \mathrm{mz}$ como una manera de integrar un sistema de producción amigable al medio ambiente en un área protegida con alto potencial productivo. La producción de queso suizo inició a pequeña escala en el 1998 cuando un experto suizo dio capacitación en nuevas técnicas para la elaboración de queso suizo maduro y la cooperación española apoyó en el acondicionamiento de un local de procesamiento y empaque de queso.
Esta Asociación se encuentra ubicada en san Nicolás municipio de Estelí, departamento de Estelí en el km 135 Carretera panamericana.

En la actualidad ASOPASN cuenta con 55 socios y 14 trabajadores que se encuentran distribuidos en los diferentes proyectos que como asociación llevan a cabo dentro de los cuales citamos:

\begin{tabular}{|l|l|}
\hline Áreas & $\mathbf{N}^{\mathbf{l}}$ de Trabajadores \\
\hline Producción de queso maduro & 1 Trabajador \\
\hline Ganado mayor y menor & 1 Trabajador \\
\hline Área de cuido de cabras & 1 Trabajador \\
\hline Café Orgánico & 1 Trabajador \\
\hline Hortaliza Orgánica & 2 Trabajadores \\
\hline Lombricultura & 1 Trabajador \\
\hline Turismo Rural & 2 Trabajadores \\
\hline Guía Turístico & 1 Trabajador \\
\hline Área de Plantas Medicinales & 1 Trabajador \\
\hline Semillero & 1 Trabajador \\
\hline Actividades Múltiples & 1 Trabajador \\
\hline Proyectos, Administración & 1 Trabajador \\
\hline TOTAL & 14 Trabajadores \\
\hline
\end{tabular}

\section{Misión, Visión, Valores Y Estructura Organizativa De ASOPASN}

Misión: Somos una Finca Agro Turística promoviendo el Desarrollo Integral de la comunidad, y Brindando servicios como: Alojamientos, Senderismo, Tours por las diferentes Áreas, Alimentación, Capacitaciones y Artesanías

Visión: Ser una Finca Agro Turística que promueva un Ambiente Humano, y acogedor en Armonía con la Naturaleza, potencializando el desarrollo, por y para la comunidad, conservando nuestra cultura.

\section{Valores}

- Conservación, y Protección, del Medio Ambiente

- Promoción de la Agricultura Orgánica

- Producción de Alimentos Sanos

- Comercialización Justa 
- Seguridad del Huésped

- Honestidad

- Organización Comunitaria

\section{Proceso de Administración:}

Como toda empresa legalmente constituida la asociación de programas agrícolas realiza la planeación, dirección, control y organización este proceso administrativo es desarrollado por una sola persona que realiza lo que es la parte contable de toda la asociación.

El proceso de planeación y organización lo llevan a cabo mediante reuniones que se realizan cada lunes, en dicha reunión se trata aspectos importantes como lo son las estrategias que contribuyan a un buen funcionamiento de la asociación.

\section{Estrategias utilizadas:}

- Mejorar la calidad: Este aspecto lo implementan atráves de los insumos utilizados en lo que respecta al procesamiento de la leche para la elaboración del queso, insumos como cuajo en líquido, cultivo de bacterias, salmuera, entre otros.

- Excelentes precios: Esta estrategia les ha resultado muy factible ya que esta asociación cuenta con un mercado diferenciado con buen poder adquisitivo que estaría dispuestos a pagar cualquier cantidad por consumir este queso de excelente calidad.

- Mejorar la gestión comercial

- Aumentar las coberturas de mercados: Esta asociación pese alas limitantes de comercialización que tienen a causa del vencimiento del registro sanitario, se han dado a la tarea de buscar nuevos lugares para ofrecer sus productos, tal es el caso de la participación de estos en las ferias realizadas los viernes en el parque central de Estelí, también está la distribución de queso por pedidos que se les realiza.

- Mejorar el servicio del cliente: Esta estrategia es de vital importancia para esta asociación, ya que además de ofrecer quesos, de igual forma prestan otros servicios como los turísticos que hacen que los visitantes queden encantados de sus productos y de la reserva natural.

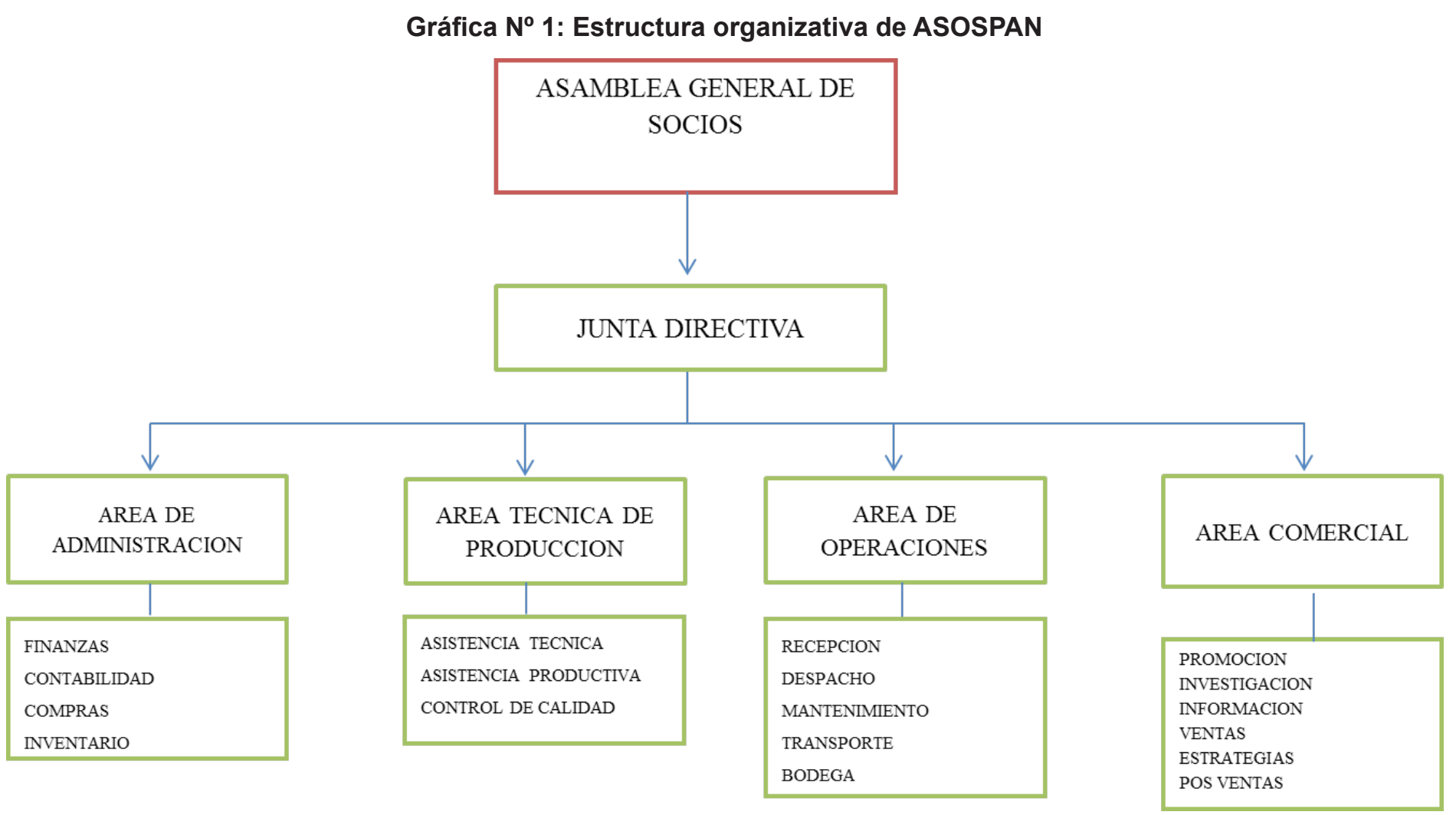


1. La Asamblea General: El órgano supremo que toma las decisiones importantes. En la actualidad esta asamblea cuenta con 55 socios de comunidades del municipio de San Nicolás.

2. Aréa de Administración: Esta constituye una de las áreas más importante en ASOPASN ya que aparte de llevar a cabo lo relacionado con el proceso administrativo, de igual forma cumple actividades propias de la contabilidad destacándose entre ellas la elaboración de estados financieros, la realización de las compras de los insumos necesarios para la producción de los diversos productos ofrecidos por la tienda incluyendo el queso, además realiza la función del control de inventario a fin de determinar la posibilidad de cubrir la demanda de quesos.

3. Área Técnica de Producción: Dentro de esta área se llevan a cabo funciones vinculadas al proceso productivo de los productos, como lo es el caso de la asistencia técnica, productiva y sobretodo el control de calidad para cumplir con las exigencias del ministerio de la salud (MINSA).
4. Aréa de Operaciones: A como su nombre lo indica esta área se encarga de los procesos de operación realizados por Asopasn a fin de mantener el funcionamiento deseado, realizando funciones como la recepción de la materia prima, el mantenimiento de los productos, y el transporte vinculado a la distribución de los mismos.

5. Aréa Comercial: Esta área tiene a su cargo la responsabilidad de llevar el producto final a los intermediarios o ya sea a los consumidores finales, esto mediante lo medios de promoción e información donde se da a conocer las cualidades deseables de los productos ofertados. De igual forma desarrollan las estrategias necesarias para mejorar sus canales de comercialización o sus productos, más que todo se preocupa por identificar debilidades para en base a esto desarrollar estrategias que fortalezcan sus oportunidades de extensión.

\section{Proceso de Producción:}

Para la producción de queso se necesitan: Leche, cultivo lácteo, sal y cuajo líquido

Gráfica $N^{\circ}$ 2: Diagrama de flujo del proceso productivo de queso con método suizo

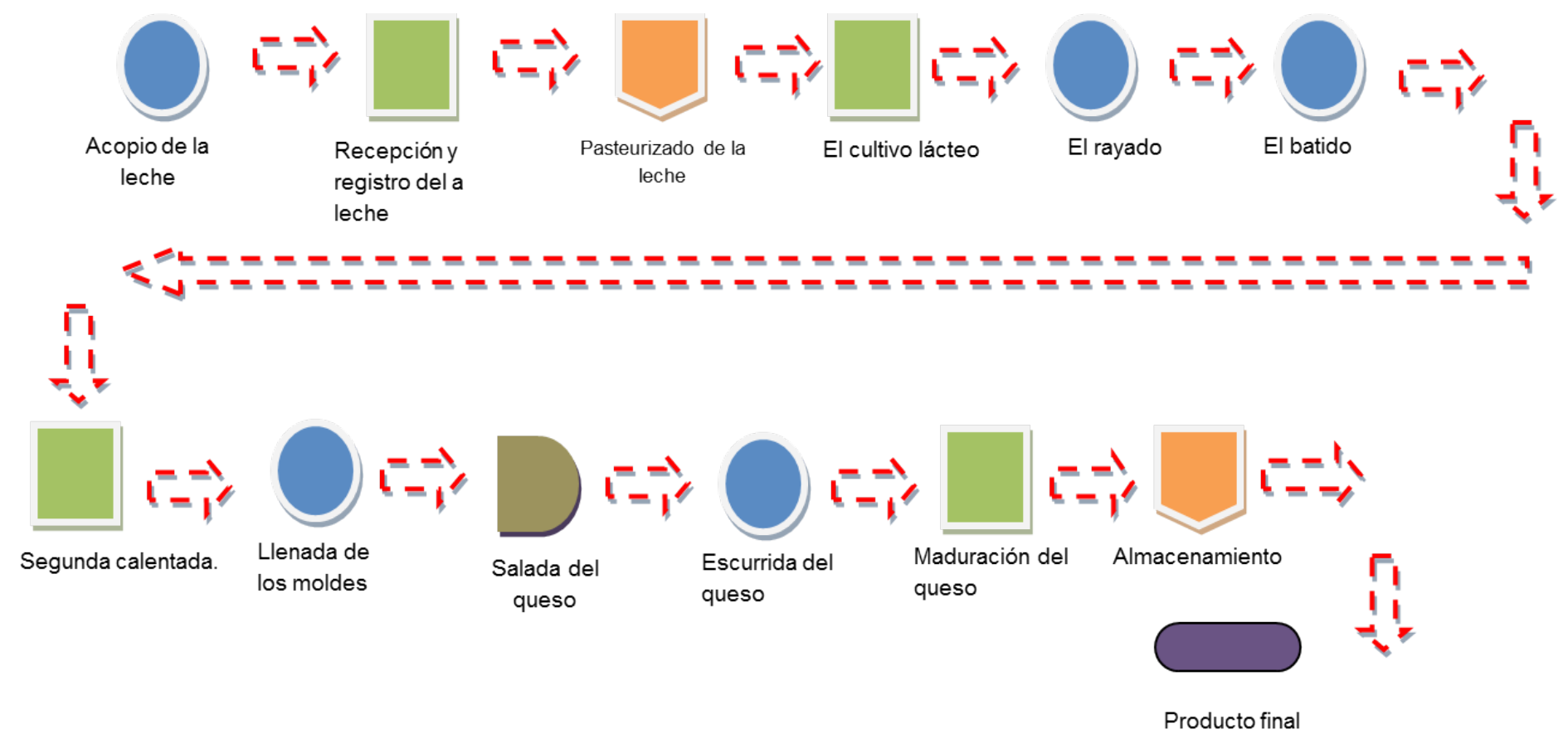




\section{Paso 1. Acopio de la leche}

En la sala de acopio se recibe la leche y se cuela para evitar que lleve suciedad. La leche primero se cuela con un colador y luego con un filtro de papel.

\section{Paso 2. Pasteurizado de la leche.}

La pasterización consiste en calentar la leche a $70^{\circ}$ grados de temperatura, durante media hora y después bajar esa temperatura a unos 35 grados.

Para subirle la temperatura la leche se pone al fuego y para bajar la temperatura, el perol se sienta en agua fría por unos 15 minutos. En esta subida y bajada de temperatura los microbios se mueren y la leche queda libre de ellos, sin estar hervida.

\section{Paso 3. El cultivo lácteo}

Una vez pasterizada la leche, se le agrega cultivos lácteos, se le agrega un gramo por cada 10 litros de leche. Se le aplica una cucharadita de cuajo líquido por cada 10 litros de leche. Estos productos los usamos en vez del cuajo tradicional, porque ya vienen graduados y el queso siempre queda igual. La leche se deja reposar media hora mientras cuaja. Los quesos maduran con el tiempo.

\section{Paso 4. La rayada}

Una vez que la leche se le agrega el cultivo lácteo se procede a la rayada o quebrada que se realiza con un instrumento llamado lira.

\section{Paso 5. El batido}

La leche se bate para que se vuelva sólida y ayude en la maduración del queso.

\section{Paso 6. Segunda calentada}

Después de este proceso se calienta por segunda vez a 40 grados. Si son 50 litros, se calienta por 18 minutos. Si son 35 litros, se calienta por 12 minutos.

\section{Paso 7. La llenada de los moldes}

Se llena el molde y se prensa durante cinco minutos, de esta manera el suero sale. El queso se saca a los 10 minutos y se le da vuelta en el molde para que las dos caras del queso queden iguales. De ahí en adelante cada hora se le da vuelta al queso, hasta que sea de noche.

\section{Paso 8. La salada del queso}

Los quesos se salan en salmuera para que el sabor quede parejo y no tan salado. La salada se hace al día siguiente de estar los quesos en los moldes. Se prepara el agua con sal y se calienta a 80 grados, en esa agua ya en reposo se meten los quesos.

El tiempo que se deja un queso en salmuera depende de su tamaño, un queso de libra o libra y media se deja durante dos horas. Un queso de 2 libras se deja cuatro horas. Uno de 10 libras diez horas. Después de pasado este tiempo, los quesos se sacan y se dejan en reposo. Durante tres o cuatro días los quesos se ponen en la salmuera. Esta agua con sal, se cuela diario para que no quede pedacitos de queso.

\section{Paso 9. La escurrida del queso}

Los quesos se ponen a escurrir tres días, así se orean y les sale la humedad.

\section{Paso 10. La madurada del queso}

Una vez los quesos están escurridos, pasan a la sala de maduración que está en el sótano de la, ahí la temperatura se mantiene fresca a unos 23 grados. La sala está limpia y las ventanas están forradas con cedazo para que pase el aire y no entre ni una mosca. En los estantes de madera los quesos puede pasar meses, los de leche de vaca con dos meses de maduración ya se pueden vender, los de cabra con 50 días ya están en su punto.

Volúmenes de Producción e Ingresos por venta de ASOPASN 
En la gráfica No.1 se puede apreciar los niveles de producción de queso de cabra y vaca durante los meses de enero a junio, correspondiente al I semestre del año 2013. En el mes de abril la producción de queso de cabra es baja en relación al mes de mayor producción que es Junio ya que esta forma parte de la estación de invierno en la cual las vacas tienden a producir mayor cantidad de leche.

Gráfica № 3: Producción de queso de cabra y vaca

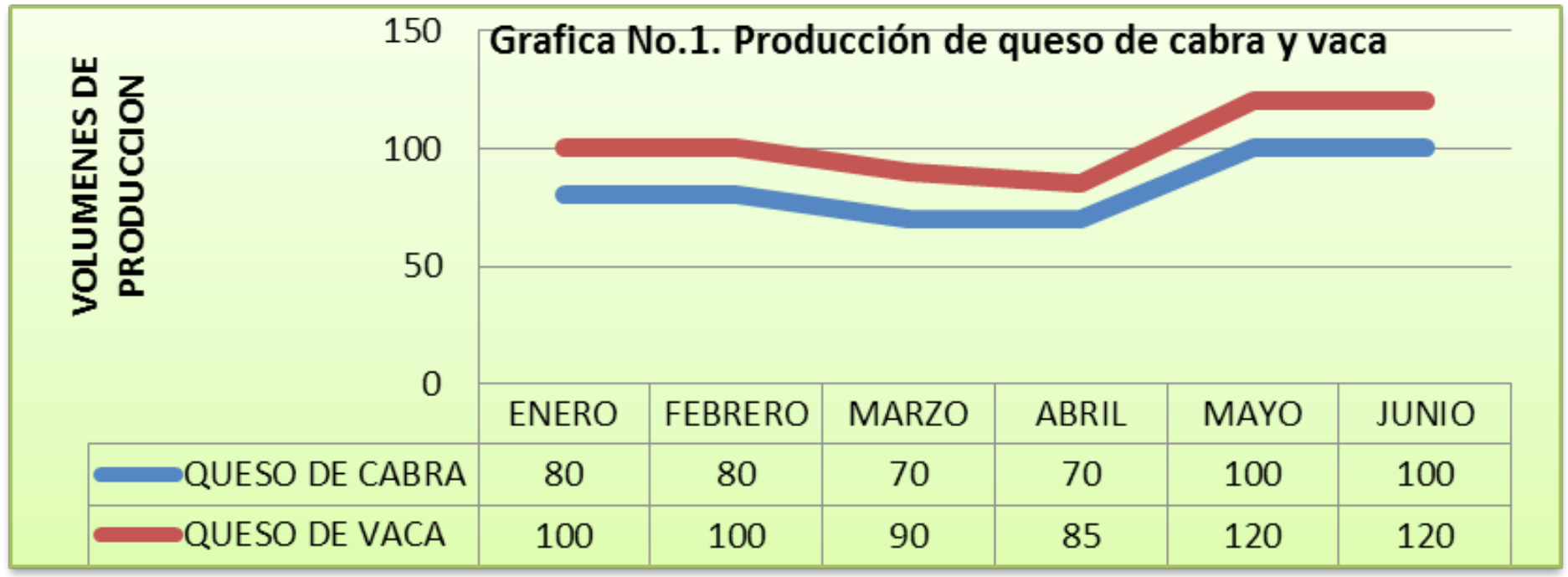

De igual forma con respecto al queso de vaca se observa que en el mes de abril se da una baja en la producción, como consecuencia de la poca leche acopiada por la estación de verano, a diferencia del mes de junio que por ser de estación de invierno la cantidad de leche producida por las vaca es mayor lo que provoca una mayor producción de queso.

\section{Gráfica N 4: Ingresos por ventas semanal}

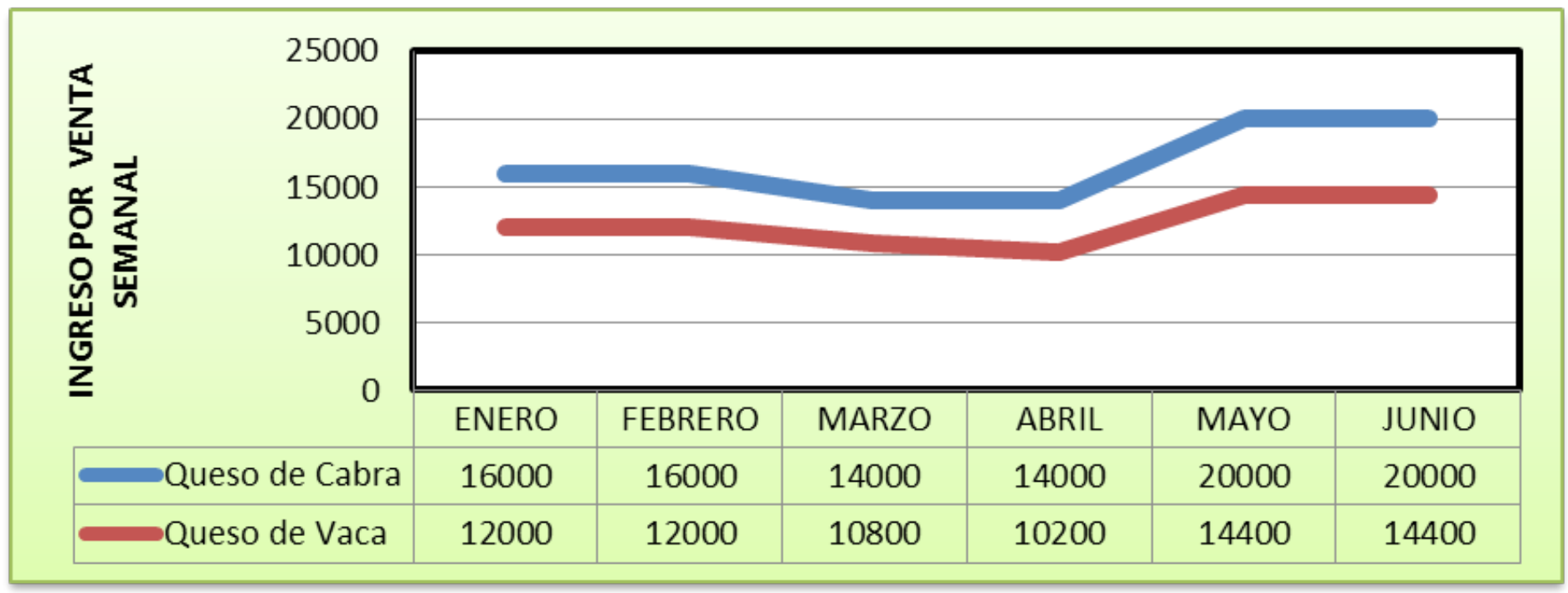


En esta gráfica se puede observar los ingresos por ventas obtenidos durante los meses de enero a junio, esto en base a los niveles de producción y al costo unitario de cada tipo de queso, dando como resultado que en el mes de marzo se obtienen menos utilidades a consecuencia de la poca producción por la época de verano, esto en comparación a los meses de mayo $y$ junio en donde se obtienen mayores ingresos a causa de la época de invierno y a la mayor producción de queso de cabra que hubo.

Así mismo se observa que hubo menos ingresos en el mes de abril en comparación a los meses de mayo y junio en donde se obtuvieron mayores ingresos en lo que respecta a la venta de queso de vaca.

De todo lo anterior se puede deducir que los ingresos por la venta de queso están en dependencia a los niveles de producción que a su vez depende en gran medida de las épocas del tiempo, ya que este hace que varíen la cantidad de leche acopiada.

Proceso de Comercialización:

Con la promoción, divulgación, participación en ferias y giras de intercambios, la asociación ha logrado posicionar los quesos y a su vez aumentar la demanda. Los materiales de promoción como las tarjetas y los brochures han ayudado mucho. Actualmente hay mercados definidos a nivel local, regional, nacional y con los turistas extranjeros quienes aprecian mucho los productos de La Garnacha. La promoción ha tenido un efecto multiplicador con recientes reportajes publicados en diario Nacionales como es La PRENSA y la revista ZONA CENTRO.

El siguiente flujo muestra el proceso de Comercialización de la Tienda La Garnacha:

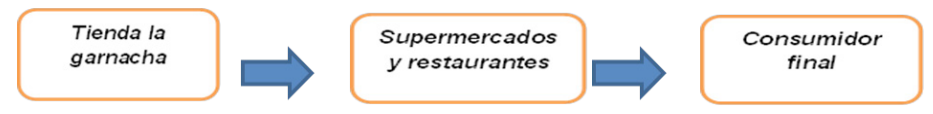

Como podemos observar se utilizan intermediarios como son los restaurantes y los supermercados, donde posteriormente llegara al consumidor final que en su mayoría son personas extranjeras, ya que los lugares en los que comercializa son turísticos de mucha afluencia de gente europea y estadounidense.

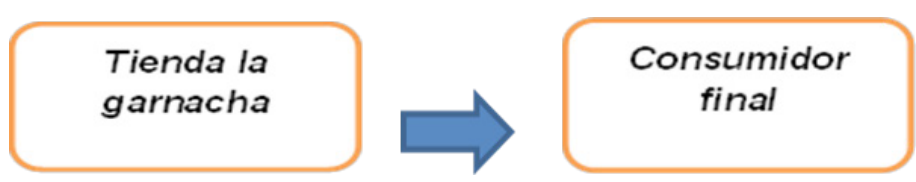

El queso, se ofrece directamente en tienda la Garnacha donde los compradores asisten de manera directa y obtienen el producto, también se oferta todos los viernes en la Feria de Mercadito verde, en donde los clientes tienen la oportunidad de adquirirlo un precio accesible

\section{Lugares de venta de Queso:}

\begin{tabular}{|l|l|}
\hline \multicolumn{2}{|c|}{ Lugares de venta } \\
\hline \multirow{4}{*}{ Managua } & Babaria Delikatess \\
\cline { 2 - 2 } & La Ola Verde \\
\cline { 2 - 2 } & Tininiska cooperativa \\
\cline { 2 - 2 } & Taska de kiko \\
\hline \multirow{2}{*}{ León } & Panadería Pan y Paz \\
\cline { 2 - 2 } & Barca de oro \\
\hline \multirow{4}{*}{ Estelí } & Mercado verde de Estelí \\
\cline { 2 - 2 } & Tienda Garnacha \\
\cline { 2 - 2 } & Súper Las Segovia \\
\hline
\end{tabular}

\section{Competencia}

En este caso tan importante la competencia a nivel local es casi nula, pesé a que existen muchas empresas grandes y pequeñas que trabajan en el sector lácteo, pero en esta parte entra en juego las estrategias de publicidad y la elaboración de queso atráves del innovador método suizo a como lo afirma pablo centeno

"Hay mucha competencia en el mercado con el queso de vaca, pero el nuestro tiene un sabor único debido a las técnica suizas" comentó pablo centeno responsable de producción" 
Como Asociación están conscientes de que tiene competencia en lo que respecta a la producción de queso de vaca, pero ellos ofrecen diversidad mediante la elaboración de queso de cabra, que se ha convertido en uno de los mayores atractivos de este centro turístico. Pese a lo anterior, a esta asociación les complace en ser los únicos productores de queso suizo de la zona norte de Estelí, por lo cual su competencia disminuye en gran medida.

\section{Estándares de calidad exigidos por el Ministerio de Salud}

En vista de conocer sobre la situación actual de ASOPASN en cuanto al registro sanitario, se incorporó un nuevo instrumento de obtención de información dirigido a la Sra. Egda Torres responsable de coordinación de programas de sistemas locales de atención integral de salud (SILAIS) Estelí la cual nos comentó:

El registro sanitario es el acto administrativo mediante el cual el MINSA evalúa y certifica un alimento procesado, conforme las normas y reglamento de inocuidad alimentaria o bien es el Aval sanitario que otorga el MINSA a los productos que cumplen con las normas sanitarias de inocuidad y calidad (Sra.Egda Torres coordinadora de programas SILAIS Estelí).

\section{Requisitos para la Obtención del Registro} Sanitario:

- Llenar la solicitud de registro sanitario, por tipo de producto valor de la solicitud $\mathrm{C} \$ 50.00$.

- Ficha técnica del producto a registrar

- Presentar 3 muestras de 5000 g cada un apara solidos o 3 litros para líquidos, del producto que desea registrar

- Copia de la licencia sanitaria vigente para el establecimiento de producción nacional o de la bodega para productos fabricados en el extranjero.

- Certificado de libre venta para productos importados de terceros países.

- Etiqueta original para los productos importados y nacionales que ya estén en el mercado. Proyecto de etiquetas para nuevos productos.

- Comprobante de pago de (análisis).

- Comprobante de pago del certificado. C\$500.00

\section{Vigencia:}

El registro sanitario tiene una vigencia de 5 años

\section{Mecanismo para Registro:}

- El interesado presenta ante la autoridad sanitaria los requisitos completamente establecidos.

- La autoridad sanitaria verifica el cumplimiento de la documentación presentada.

- La autoridad sanitaria ingresa los expedientes con documentación completa.

- Remisión de las muestras de laboratorio, para su respectivo análisis.

- Como constancia de que un producto ha sido registrado, la autoridad sanitaria extenderá una certificación en la que consta el número del Registro Sanitario.

- A los productores de un mismo fabricante, que tienen la mismas formula y que varían únicamente su fórmula, presentación, nombre o marca, se le asignara un único número de registro.

\section{Requisitos de Inscripción Sanitaria:}

- Llenar la solicitud de inscripción sanitaria, por tipo de producto y tiene un valor de C $\$ 50.00$.

- Copia vigente de la licenciatura sanitaria de la bodega de Almacenamiento del producto.

- Presentar 3 muestras de 500 gramos para cada uno de los sólidos o 3 litros para líquidos, del producto que se desea inscribir.

- Comprobante de pagos (análisis)

- Comprobante de pago de certificación de inscripción sanitaria ( $\mathrm{C} \$ 500.00)$.

\section{Licencia Sanitaria:}

La licencia sanitaria es la autorización que extiende el MINSA atráves de los SILAIS (Sistemas Locales de Atención Integral en Salud), a todos aquellos 
establecimientos de alimentos que cumplan con las normativas sanitarias establecidas. Para el establecimiento es importante porque demuestra a los consumidores, que es un local que está regulado por el órgano rector de la salud en nuestro país (MINSA).

\section{Requisitos:}

- Solicitud por escrito

- Autorización para ubicación y construcción del establecimiento otorgada por la autoridad competente.

- Cumplir con los requisitos establecidos en material ambiental de acuerdo a la legislación nacional.

- Croquis y distribución del establecimiento.

\section{Líneas estratégicas para mejorar la comercialización de queso en ASOPASN}

Línea N 1: Búsqueda de Financiamiento para mejorar la Infraestructura actual.

Línea $\mathbf{N}^{\circ}$ 2: Cumplir con las normativas y condiciones requeridas por el Ministerio de Salud.

Línea $N^{\circ}$ 3: Localización de nuevos acopios de leche de cabra

Línea 4: Incrementar los niveles de Producción de queso

Línea $\mathbf{N}^{\circ}$ 5: Creación de un establo que preste condiciones de calidez necesaria para aumentar la producción de leche de cabra.

Línea $\mathbf{N}^{\circ}$ 6: Fijación de precio en base al costo de producción

\section{CONCLUSIONES}

Al finalizar el presente estudio investigativo en la organización que fue nuestro objetivo como lo es la Asociación de Programas Agrícolas de San Nicolás (ASOPASN) podemos concluir en base a los objetivos planteado lo siguiente:

El proceso administrativo existente en esta organización presenta debilidades en lo que respecta al seguimiento continuo necesario para el funcionamiento eficiente de cualquier empresa, esto a consecuencia a que los procesos pertenecientes a la administración solo se dan una vez a la semana ya que la administradora en funciones argumenta que el salario que devenga es muy inferior, y esto no le permite cubrir los gastos en los que pudiese incurrir al viajar todos los días. Por lo antes mencionados podemos determinar que las estrategias utilizadas carecen de planificación, organización, dirección y sobretodo de control de actividades en la organización.

En lo que respecta al proceso productivo encontramos un magnifico proceso de elaboración de queso atráves del método suizo, que ofrece una variedad de sabor y la innovación en productos. De igual forma se encuentra la limitante de la infraestructura del área de elaboración del queso ya que estas se encuentra transversas en comparación a lo que exige el MINSA.; según las exigencias de estos las áreas deben mantener el siguiente orden Recepción de la leche, área de procesamiento de la leche, área de salado, área de ubicación del queso en moldes y por último el área de almacenamiento.

Lamentablemente en ASOPASN las áreas tienen otro orden, primero se encuentran el área de recepción, área de salado de queso, el área de ubicación del queso en los moldes, luego el área de procesamiento de la leche y por último la bodega; dicho error hace que ellos expongan el queso al aire libre para poder llevarlo a la bodega de maduración lo cual fue motivo suficiente para negarles el derecho de renovar el registro sanitario argumentando de que el queso se encuentra expuesto a los contaminantes que se encuentran en el ambiente.

De acuerdo con el proceso de comercialización se analizaron los intermediarios utilizados por ASOPASN y evaluamos a estos como empresas reconocidas como lo es el supermercado las Segovias, restaurantes con propietarios nacionales e internacionales lo que nos permite concluir que tienen un gran nivel de 
aceptación de sus productos. Además de participar cada viernes en ferias las cuales se desarrollan en el parque central de Estelí.

Pero este proceso se ha visto limitado por el vencimiento del registro sanitario ya que a raíz de esto se les han cerrado las posibilidades de distribuir su producto en otros establecimientos como lo es el caso del supermercado la colonia que discontinuo la compra del queso a consecuencia de este vencimiento. Así mismo existe un gran desinterés por parte de los socios de ASOPASN en cuanto a cumplir con los requisitos exigidos por el ministerio de la salud, ya que ellos han descontinuado sus visitas a este local en Managua, por lo cual es notorio que existe una gran falta de visión empresarial.

En resumen podemos determinar que existen grandes oportunidades en el proceso productivo y de comercialización que ayudaran al crecimiento de esta asociación las cuales necesitan ser aprovechadas y solo se podrá lograr si se actúa con mayor visión y la implementación de estrategias empresariales que les ayude a obtener el éxito de la asociación.

\section{BIBLIOGRAFÍA}

Vásquez, Janete.2008.Importancia de la Ley No. 645 (Ley MIPYME), para las empresas. Sitio web: http://www.caconic.org.ni/dwnlds/articulos/leymipyme.pdf.

Morán, (2007) Guía de observación para auxiliares de investigación. Sitio web: <http://www.eumed. net/ce/2007b/jlm.htm>

Martínez, J.D. (2008, junio12) La entrevista como instrumento de investigación. El Nuevo Diario [En línea], en español. Disponible: http://www. elnuevodiario.com.do/app/article.aspx?id=106667

Barquero Incer, Jaime.(2009).Sistemas de comercialización. Sitio web:http://www. mercados.us/inancieros/capitales/segmentacion/ comercilaizacion y mercado.
Rivas, Christian. (2008).Estrategias de Marketing y comercialización. Sitio web: http://magetiono. com/artículos/estrategias de marketing y comercialización .html

Fong. Ernesto.(2009). Tipos de comercialización Sitio web:http://www.mercados.us/financiero/capitales/ segmentaion/comercializa-ción y mercado.

Valga, Juan Carlos.2010. Realidad Pyme en Nicaragua. Sitio web: http://jcvalda.wordpress. com/2010/06/18/realidad-pyme-en-nicaragua/

Vásquez, Janete.2008.Importancia de la Ley No. 645 (Ley Mipyme), para las empresas. Sitio web: http:// www.caconic.org.ni/dwnlds/articulos/ley-mipyme. pdf

Gaitán, Fabio, José. (2009). Folleto de la clase Administración I. UNAN-FAREM, ESTELI.

González .R. Orbelina. Folleto dela clase Control Gerencial. FAREM-ESTELI

Gómez. García. Reynaldo (2011). Folleto de la clase. Macroeconomía. FAREM-ESTELI

Maradiaga, M.A (2011).Formulación y Evaluación de Proyecto (1ra Ed).Managua, Nicaragua.

Coventry, Pedro, Tomas Albert.(2000). Desarrollo de la comercialización Agrícola, 5ta. Edición.

Juran, J. M,; Gryna,Jr FranCm, Bingham, Jr R.S(2005). Manual de control de calidad [En línea, Volumen 1, segunda Edición.

Rizo, Sevilla Alberto (2010). Folleto de la clase Mercadotecnia Internacional. UNAN-FAREM, ESTELI 